\title{
Performance of an ATM LAN switch with back-pressure function
}

\author{
Hiroyuki Ohsaki, Naoki Wakamiya, Masayuki Murata and Hideo Miyahara \\ Department of Information and Computer Sciences \\ Faculty of Engineering Science, Osaka University \\ 1-3 Machikaneyama, Toyonaka, Osaka 560, Japan \\ (Phone) +81-6-850-6588 \\ (Fax) +81-6-850-6589 \\ (E-mail)oosaki@ics.es.osaka-u.ac.jp
}

\begin{abstract}
Traffic control schemes for ATM networks can be classified into two categories: reactive congestion control and preventive congestion control. Reactive congestion controlcan be effective in ATM local area networks as well as preventive congestion control. A possible scheme to realize efficient reactive congestion control is a switch architecture, which possesses buffers on both sides of input and output ports with a back-pressure function. Especially, when this switch is applied to ATM LANs for data transfer services, its performance should be evaluated by taking into account the bursty traffic, which is a main purpose of the current paper. In this paper, we show the maximum throughput of such an ATM switch with back-pressure function under bursty traffic through an analytic method. In addition to a balanced traffic condition, unbalanced traffic and a mixture of bursty and stream traffic are also considered. Through numerical examples, we show the effects of the average packet length and the output buffer size on the perfomance of the switch quantitively.
\end{abstract}

\section{Keywords}

ATM LAN, input and output buffer switch, back-pressure function, bursty traffic

\section{INTRODUCTION}

An ATM (Asynchronous Transfer Mode) technology realizes B-ISDN (Broadband Integrated Services Digital Network) by asynchronously treating various multimedia information such as data, voice and video. The benefit of the ATM technique is enjoyed by a statistical multiplexing of multimedia traffic by dividing it into fixed size packets (called cells). Much efforts of researches, developments and standardizations have been extensively devoted to public wide area ATM networks. In addition, the ATM technology also seems to be promising for realization of 
new high speed local area networks (LANs) to cope with a rapid advance of high-speed and multimedia-oriented computers.

Traffic control is an important issue for an efficient utilization of network resources in an ATM based network including wide and local area networks. Traffic control schemes can be classified into two categories; reactive congestion control and preventive congestion control. The reactive congestion control is the way to resolve network congestion after its occurrence. The preventive congestion control is, on the contrary, to prevent a network from its falling into congestion. The latter is now widely recognized as an effective way in wide area networks since the propagation delay is not negligible and QOS (Quality Of Service) requirements should be preserved in a strict manner. In ATM LANs, however, the propagation delay is small and is used in a private environment. Hence, preventive congestion control becomes meaningful because of its easier implementation.

To implement preventive congestion control in ATM LANs, Fan et al. recently proposes a switch architecture which possesses buffers on both sides of input and output ports with a backpressure function [Ili92a]. The back-pressure function is provided to avoid a temporary congestion by prohibiting transmission of cells from input buffer to output buffer when the number of cells in output buffer exceeds a some threshold value. The performance of this kind of the switch has been analyzed by Iliadis in [Ili92a, Ili90, Ili92b]. However, he assumed that interarrival times of cells at each input port follow a geometric distribution. Especially when the above switch is applied to ATM LANs for supporting data transfer service, its performance should be evaluated by taking into account the bursty nature of arriving traffic, i.e., packets coming from the upper protocol layers. In this paper, we show the performance of an ATM LAN switch with back-pressure function against bursty traffic, that is, the continuously arriving cells (forming a packet) which are destined for the same output port are treated for the analysis.

This paper is organized as follows. In Section 2, an analytic model of the ATM switch we will evaluate is described. In Section 3, the steady state probability of our model is derived. In Section 4, the maximum throughput is derived based on the results of Section 3. Our subjects of investigation are extended to an imbalance traffic at input and output ports, and a mixture with stream traffic as well. Finally, in Section 5, we conclude our paper with some remarks.

\section{ANALYTIC MODEL}

In this section, we describe an ATM LAN switch with back-pressure function followed by an introduction of our analytic model. The number of input ports (and output ports) is assumed to be $N$. Our ATM switch is equipped with buffers at both sides of input and output ports (see Figure 1), and the buffer sizes are defined as $N_{I}$ and $N_{O}$, respectively. The switching speed of cells from input buffer to output buffer is $N$ times faster than the link speed, that is, at most $N$ cells may be transferred from input buffer to output buffer in a time slot. It is called the backpressure function to prohibit transmission of cells from input buffer to output buffer by signaling back from output buffer to input buffer when the number of cells in output buffer exceeds a some predefined threshold value [RFM94]. By this control, a cell overflow at output buffer can be avoided (see Figure 2). However, it introduces HOL (Head Of Line) blocking of cells at input buffer, which results in the limitation of the switch performance.

We assume that a stream of successively arriving cells forms a packet, and the number of cells in the packet follows a geometric distribution with mean $\overline{B L}$. Let $p$ denote the probability that 


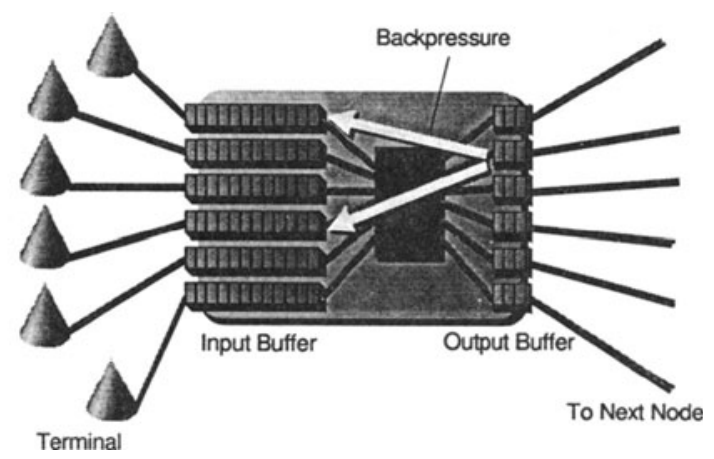

Figure 1 The ATM switch with back-pressure function.

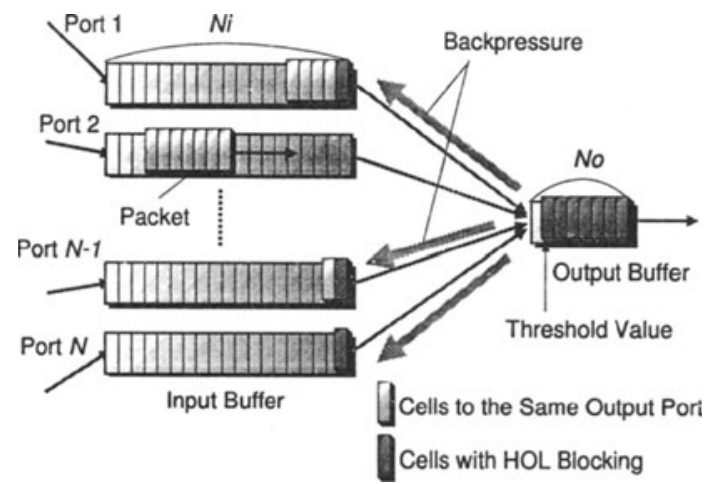

Figure 2 The analytic model.

a newly arriving cells belong to the same packet which is arriving at same input port. Thus, we have a relation;

$\overline{B L}=\sum_{i=1}^{\infty} i(1-p) p^{i-1}=\frac{1}{1-p}$.

We will assume that all cells are stored under first-in-and-first-out (FIFO) discipline at input buffer.

The practical threshold value at output buffer would be $N_{O}-N$ as proposed in [RFM94]. However, as an ideal case, we assume that the HOL cells are transferred from input buffer to 
output buffer at random until the output buffer is filled up. In other words, when the output buffer is fully occupied with cells, input buffers which have HOL cells destined for that output buffer receives the back-pressure signal to stop cell transmission. Then, all HOL cells are awaited at the head of each input buffer. As soon as the cell in output buffer are transmitted onto the output link, one of HOL cells is selected at random and transmitted to the output buffer. Therefore, it is considered that HOL cells destined for the same output port form a virtual queue which we will call a HOL queue. While HOL cells are actually stored at the HOL queue, it can be regarded that HOL packets form the HOL queue in our modeling. Therefore, in what follows, we will use "HOL cell" and "HOL packet" without discrimination.

The switch size $N$ will be assumed to be infinity in the following analysis. By introducing the assumption of the infinite switch size, we can focus on one single output port and its associated HOL queue. The infinite switch size gives the performance limitation as shown in [Ili90, KHM86]. For example, when compared with the finite case, the maximum throughput with the infinite case gives an upper limit. Further, it is known that the close value are obtained when $N$ reaches at 16 or 32 when the cell interarrivals follow a geometric distribution [Ili90]. In this paper, we will examine this fact in the case of bursty traffic in Section 4.

In this paper, we will first assume the capacity of the input buffer $N_{I}$ to be infinity in obtaining the maximum throughput and the packet delay distribution. This assumption is realistic because the memory speed of the output buffer should be $N$ times faster than the link speed. Thus, the capacity of the output buffer should be limited. On the other hand, the input buffer can be operated at the same speed as the link, which results in that the large capacity can be equipped. In what follows, we consider a discrete time system in which its slot time equals to a cell transmission time on the input/output link.

Under assumptions described in the above, the system state is represented by two random variables $Q_{k}$ and $H_{k}$, where $Q_{k}$ is the number of cells at some output buffer and $H_{k}$ is the number of HOL cells at input buffers associated with that output buffer, respectively. In the next section, the steady state probability of the doublet of two random variables $\left(Q_{k}, H_{k}\right)$ is derived.

\section{DERIVATION OF STEADY STATE PROBABILITY}

In the following sections, we focus on a single output port and its associated HOL queue without loss of generality. Let $H_{k}$ and $Q_{k}$ denote the random variables for the number of HOL cells and the number of cells in the output buffer at $k$-th slot, respectively. We further introduce $A_{k}$ for a random variable to represent the number of HOL packets newly arriving at the HOL queue at the beginning of $k$-th slot. By defining a symbol $(x)^{+}=\max (0, x)$, we have the following equations.

1. $H_{k-1}+A_{k} \leq N_{O}-\left(Q_{k-1}-1\right)^{+}$, that is, all HOL cells can be transferred to the output port: At first, we have

$Q_{k}=\left(Q_{k-1}-1\right)^{+}+H_{k-1}+A_{k}$.

Let $B_{k}$ be the number of the HOL packets which further generate HOL cells at the current $k$-th slot. When there exist $i$ HOL packets in HOL queue, the probability that $B_{k}$ becomes $j$ 
is:

$b_{i, j}=\left(\begin{array}{c}i \\ j\end{array}\right) p^{j}(1-p)^{i-j}$

and we have

$H_{k}=B_{k}$

2. $H_{k-1}+A_{k}>N_{O}-\left(Q_{k-1}-1\right)^{+}$, that is, some HOL cells cannot be transferred to the output port at $k$-th slot:

$N_{O}-\left(Q_{k-1}-1\right)^{+}$HOL cells are transferred to the output buffer, and $C_{k}$ cells out of them further generate HOL cells in the current $k$-th slot. Therefore, $H_{k-1}+A_{k}-\left(N_{O}-\left(Q_{k-1}-1\right)^{+}\right)$ cells are kept waiting at the HOL queue. Hence,

$Q_{k}=N_{O}$

$H_{k}=H_{k-1}+A_{k}-\left(N_{O}-\left(Q_{k-1}-1\right)^{+}\right)+C_{k}$.

Since the switch size $N$ is assumed to be infinity, arrivals of packets at input ports in time slot are assumed to follow a Poisson distribution [KHM86]. Therefore,

$a_{j} \equiv P[A=j]=P\left[A_{k}=j\right]=\frac{\lambda_{p}^{j} e^{-\lambda_{p}}}{j !}$.

In the above equation, $\lambda_{p}$ is the mean arrival rate of packets at each input port. By defining $\lambda_{c}$ as the mean arrival rate of cells at input ports, we have

$\lambda_{c}=\lambda_{p} \overline{B L}$

Now, we consider $s_{n, m, n^{\prime}, m^{\prime}}$, the transition probability from state $\left[Q_{k-1}=n, H_{k-1}=m\right]$ to state $\left[Q_{k}=n^{\prime}, H_{k}=m^{\prime}\right]$. The transition probability $s_{n, m, n^{\prime}, m^{\prime}}$ is obtained as follows.

1. When $n^{\prime}<N_{O}$, that is, when the back-pressure function in not utilized:

From Equation (2), we have

$A_{k}=Q_{k}-\left(Q_{k-1}-1\right)^{+}-H_{k-1}$.

When $m^{\prime}$ packets of $Q_{k}-\left(Q_{k-1}-1\right)^{+}$HOL packets further generate cells at the next slot, we have a relation

$s_{n, m, n^{\prime}, m^{\prime}}=a_{n^{\prime}-(n-1)^{+-}} b_{n^{\prime}-(n-1)^{+}, m^{\prime}}$.

2. When $n^{\prime}=N_{O}^{\prime}$, that is, when the back-pressure function is used:

From Equation (6), we have

$A_{k}=N_{O}-\left(Q_{k-1}-1\right)^{+}-H_{k-1}+\left(H_{k}-C_{k}\right)$. 
Since $C_{k}$ packets of $N_{O}-\left(Q_{k-1}-1\right)^{+}$HOL packets further generate cells at the next slot, we have a relation

$$
s_{n, m, n^{\prime}, m^{\prime}}=\sum_{i=0}^{m^{\prime}} a_{n^{\prime}-(n-1)^{+}-m+i} b_{n^{\prime}-(n-1)^{+}, m^{\prime}-i} .
$$

Once we have $s_{n, m, n^{\prime}, m^{\prime}}$, the steady state probability $r_{n, m}$,

$$
r_{n, m}=\lim _{k \rightarrow \infty} P\left[Q_{k}=n, H_{k}=m\right]=P[Q=n, H=m]
$$

is obtained from Equations (10) and (12).

1. When the state is $[Q=0, H=0]$, the output port becomes idle, i.e., we have

$$
r_{0,0}=1-\rho
$$

where $\rho$ is defined as the maximum throughput normalized by the link capacity. By our assumption that the size of the input buffer is infinity, the maximum throughput $\rho$ is equivalent to the cell arrival rate $\lambda_{c}$ in steady state if it exists.

2. By considering all states that may be changed to state $[Q=n-1, H=0]$, we have (see Figure 3)

$$
r_{n, 0}=\frac{1}{s_{n, 0, n-1,0}}\left\{r_{n-1,0}-\sum_{i=0}^{n-1} \sum_{j=0}^{i} s_{i, j, n-1,0} r_{i, j}\right\} \quad\left(0<n \leq N_{O}\right) .
$$

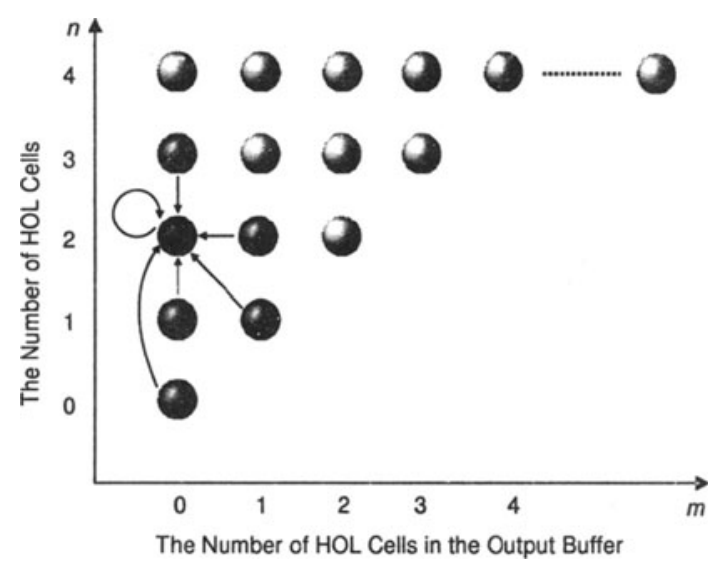

Figure 3 State transition diagram in the case of $m=0$ and $0<n \leq N_{O}$. 
3. By considering all states that may be changed to state $[Q=n, H=m]$, we have (see Figure 4)

$$
r_{n, m}=\frac{1}{1-s_{n, m, n, m}}\left\{\sum_{i=0}^{n-1} \sum_{j=0}^{i} s_{i, j, n, m} r_{i, j}+\sum_{k=0}^{m-1} s_{n, k, n, m} r_{n, k}\right\} \quad\left(0<m, n<N_{O}\right) .
$$

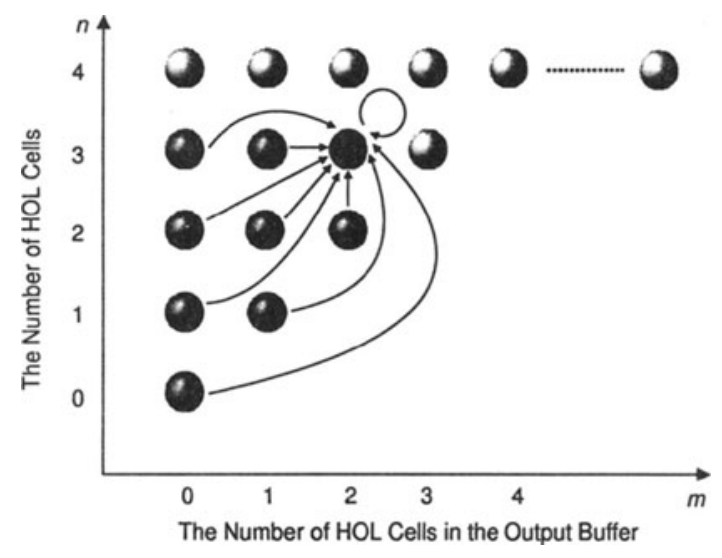

Figure 4 State transition diagram in the case of $0<m$ and $n<N_{O}$.

4. By considering all states that may be changed to the state $\left[Q=N_{O}, H=m-1\right]$, we have (see Figure 5)

$$
r_{N_{O}, m}=\frac{1}{s_{N_{O}, m, N_{O}, m-1}}\left\{r_{N_{b}, m-1}-\sum_{i=0}^{N_{b}-1} \sum_{j=0}^{i} s_{i, j, N_{b}, m-1} r_{i, j}-\sum_{k=0}^{m-1} r_{N_{b}, k}\right\} \quad(0<m) .
$$

\section{MAXIMUM THROUGHPUT ANALYSIS}

In this section, we obtain the maximum throughput using the steady state probability derived in Section 3, under balanced traffic condition in Subsection 4.1, under output unbalanced traffic condition in Subsection 4.2, and under input unbalanced traffic condition in Subsection 4.3. We will further consider the case of a mixture of bursty and stream traffic in Section 4.4.

\subsection{Case of Balanced Traffic Condition}

In this subsection, a balanced traffic condition is assumed, that is, a mean packet arrival rate at every input ports is identical and each packet determines its output port with an equal probability $1 / N$. 


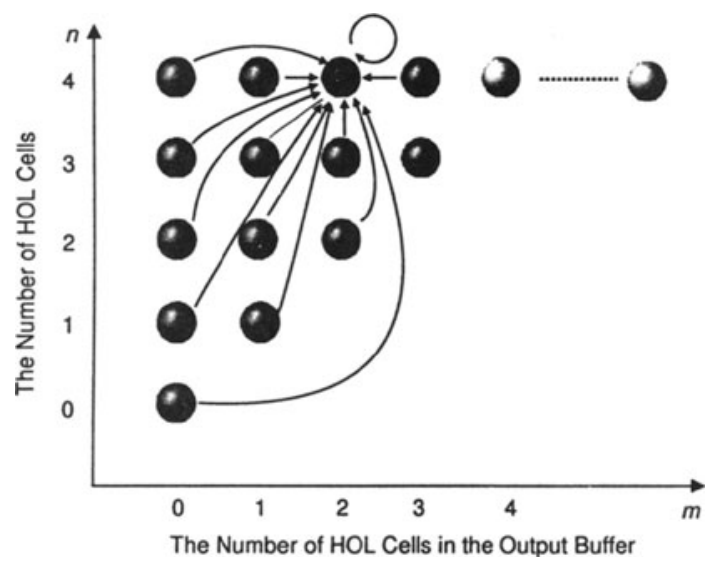

Figure 5 State transition diagram in the case of $0<m$ and $n=N_{O}$.

In order to obtain the maximum throughput of our ATM switch, we consider the case where all input ports are saturated so that packets are always waiting in HOL queues. In this case, we have

$\sum_{i=1}^{N} A^{i}=N-\sum_{i=1}^{N} H^{i}$

where $A^{i}$ is the random variable which represents the number of arriving packets destined for output port $i$ in a slot and $H^{i}$ is the random variable for the number of HOL cells destined for output port $i$. By dividing the above equation by $N$ and letting $N$ to be infinity, we have

$\lambda_{p}=1-\bar{H}$,

where $\bar{H}$ is the average number of HOL cells, and can be expressed with $r_{n, m}$ derived in Section 3 as

$\bar{H}=\sum_{n=0}^{N_{O}} \sum_{m=1}^{\infty} m r_{n, m}$

From Equations (8) and (19), we have

$\lambda_{c}=(1-\bar{H}) \overline{B L}$.

The maximum throughput $\rho$ can be obtained by substituting $\lambda_{c}$ in the above equation with $\rho$ and solving it for $\rho$. Since $\bar{H}$ depends on $\rho, \rho$ is solved iteratively by virtue of a standard iteration technique such as a bisection method [WHPV88]. 
In Figures 6 and 7, the maximum throughput $\rho$ is plotted against the average packet length $\overline{B L}$ and the output buffer size $N_{O}$, respectively. These figures show that the packet length drastically degrades the maximum throughput. Further, we may observe that the size of output buffers must be larger than the average packet length to gain a sufficient throughput.
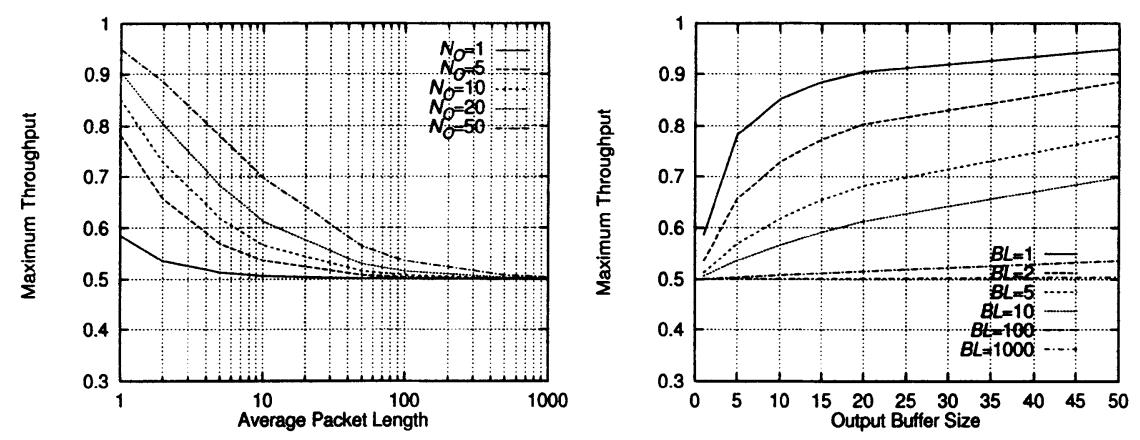

Figure 6 Maximum throughput vs. the aver- Figure 7 Maximum throughput vs. the output age packet length. buffer size.

Figure 8 shows the simulation results in the case where the switch size is finite for $N_{O}=1$ and $N_{O}=50$. The results of the analysis become slightly smaller than those of the simulation. Here, we note that the maximum throughput for $N_{O}=1$ is exactly same as a well known value of input queueing, 0.585 [KHM86].

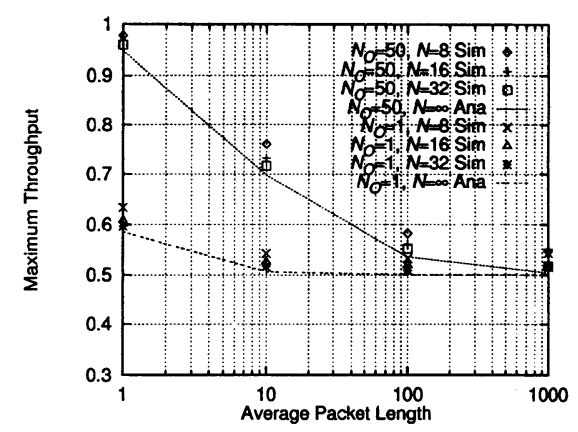

Figure 8 Comparison with simulation results.

\subsection{Case of Unbalanced Traffic at Output Ports}

In this section, output unbalanced traffic is treated following the approach presented in [Ili92a]. Output buffers are divided into two groups called $O_{1}$ and $O_{2}$. Let $q_{O}$ be a ratio of the number of 
output ports belonging to the group $O_{1}$ as

$q_{0} \equiv \frac{\left|O_{1}\right|}{N}$.

The packet arrival rate at each input port is identical. However, each packet arriving at the input port selects one of output ports in group $O_{1}$ with probability $P_{G 1}$ or one of output ports in group $\mathrm{O}_{2}$ with probability $P_{G 2}$. By assuming $P_{G 1} \geq P_{G 2}$ without loss of generality, the relative probability $r_{O}$ is denoted as

$r_{O} \equiv \frac{P_{G 1}}{P_{G 1}+P_{G 2}} \geq 0.5$

It is noted that the balanced traffic case is a special case by setting $q_{O}=0, q_{O}=1$ or $r_{O}=0.5$. Let $P_{1}$ and $P_{2}$ be the probabilities that an arriving packet is destined to an output port belonging to the $O_{1}$ and $O_{2}$, respectively, we have from Equations (22) and (23),

$$
\begin{gathered}
P_{1}=\frac{q_{O} r_{O}}{1-q_{O}-r_{O}+2 q_{O} r_{O}} \\
P_{2}=\frac{1-q_{O}-r_{O}+q_{O} r_{O}}{1-q_{O}-r_{O}+2 q_{O} r_{O}}
\end{gathered}
$$

where $\lambda_{p}$ is defined as the packet arrival rate at input ports, and $\lambda_{p 1}$ and $\lambda_{p 2}$ are the packet arrival rates at output ports belonging to the group $O_{1}$ and $O_{2}$, respectively. We then obtain

$$
\begin{aligned}
\lambda_{p 1} & =\frac{r_{O} \lambda_{p}}{1-q_{O}-r_{O}+2 q_{O} r_{O}} \\
\lambda_{p 2} & =\frac{\left(1-r_{O}\right) \lambda_{p}}{1-q_{O}-r_{O}+2 q_{O} r_{O}} .
\end{aligned}
$$

For deriving the maximum throughput, we consider a relation:

$\sum_{i=1}^{N} A^{i}=N-\left(\sum_{i=1}^{\left|O_{1}\right|} H_{1}^{i}+\sum_{i=1}^{\left|O_{2}\right|} H_{2}^{i}\right)$,

where random variables $H_{1}^{i}\left(H_{2}^{i}\right)$ is the number of HOL cells destined for the output port belonging to the group $O_{1}\left(O_{2}\right)$. By dividing the above equation by $N$ and letting $N$ to be infinity, we have

$\lambda_{p}=1-\left\{q_{o} \bar{H}_{1}+\left(1-q_{O}\right) \bar{H}_{2}\right\}$,

where $\bar{H}_{1}$ and $\bar{H}_{2}$ are the average number of HOL cells destined for the group $O_{1}$ and $O_{2}$, respectively. From Equation (8), we have

$\lambda_{c}=\left[1-\left\{q_{O} \bar{H}_{1}+\left(1-q_{O}\right) \bar{H}_{2}\right\}\right] \overline{B L}$. 
The maximum throughput $\rho$ can be obtained by substituting $\lambda_{c}$ in the above equation with $\rho$ and solving for $\rho$ in the same manner presented in Section 4.1.

In Figures 9 and 10, the relations between $\varphi_{0}$ and the maximum throughput are plotted for $\overline{B L}=1$ and $\overline{B L}=10$, respectively. These figures show that an unbalanced traffic and a larger packet size cause degradation of the maximum throughput.

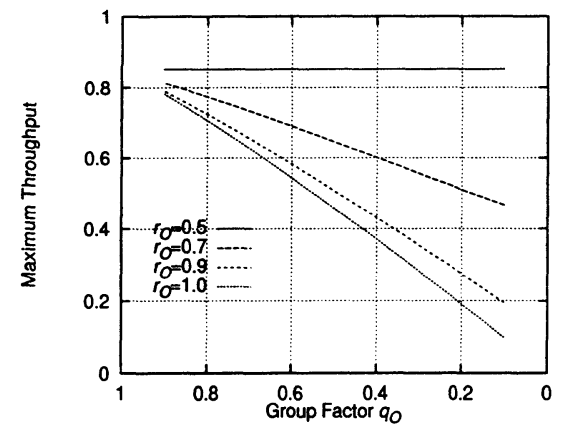

Figure 9 Unbalanced traffic at output ports $\left(N_{O}=10, \overline{B L}=1\right)$.

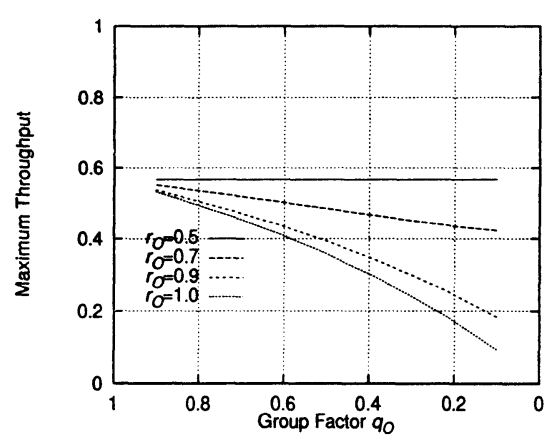

Figure 10 Unbalanced traffic at output ports $\left(N_{O}=10, \overline{B L}=10\right)$.

\subsection{Case of Unbalanced Traffic at Input Ports}

In this subsection, we evaluate the performance of the switch under the unbalanced traffic condition at the input ports. Similarly to the previous subsection, input ports are divided into two groups $I_{1}$ and $I_{2}$. Let $q_{I}$ be a ratio of the number of input ports belonging to the group $I_{1}$ defined as

$q_{I} \equiv \frac{\left|I_{1}\right|}{N}$

$\lambda_{p 1}$ and $\lambda_{p 2}$ are mean packet arrival rates at the groups $I_{1}$ and $I_{2}$, respectively. Assuming that $\lambda_{p 1} \geq \lambda_{p 2}$ is assumed without loss of generality, we introduce $r_{I}$ as

$r_{I} \equiv \frac{\lambda_{p 1}}{\lambda_{p 1}+\lambda_{p 2}} \geq 0.5$

It is noted that the balanced traffic case is the special case by setting $q_{I}=0, q_{I}=1$ or $r_{I}=0.5$. We assume that each packet arriving at the input port chooses the output port with a same probability $1 / N$. By letting $\lambda_{p}$ denote the packet arrival rate at each output port, $\lambda_{p 1}$ and $\lambda_{p 2}$ are given as

$$
\begin{aligned}
\lambda_{p 1} & =\frac{\lambda_{p} r_{I}}{1-q_{I}-r_{I}+2 q_{I} r_{I}} \\
\lambda_{p 2} & =\frac{\lambda_{p}\left(1-r_{I}\right)}{1-q_{I}-r_{I}+2 q_{I} r_{I}} .
\end{aligned}
$$


To obtain the maximum throughput, we consider the case where input ports are saturated. Recalling that we assume $\lambda_{p 1} \geq \lambda_{p 2}$, the input buffers belonging to the group $I_{1}$ is saturated first. Thus, we have a relation

$\sum_{i=1}^{\left|O_{1}\right|} A_{1}^{i}=\left|O_{1}\right|-\sum_{i=1}^{\left|O_{1}\right|} H^{i}$

where the random variable $A_{1}^{i}$ is the number of packets arriving at input port $i$ belonging to the group $I_{1}$. By dividing the above equation by $N$ and letting $N$ to be infinity, we have

$\lambda_{p 1}=1-\bar{H}$.

From Equation (8), the following relation holds:

$\lambda_{c 1}=(1-\bar{H}) \overline{B L}$,

where $\lambda_{c 1}$ is the mean packet arrival rate at each input port belonging to the group $I_{1}$. The maximum throughput $\rho$ can be obtained by substituting $\lambda_{c 1}$ in the above equation with $\rho$ and solving for $\rho$ as in the same manner presented in Subsection 4.1.

Figures 11 and 12 show the maximum throughput dependent on $q_{I}$ for $\overline{B L}=1$ and $\overline{B L}=10$, respectively. These figures show that an unbalanced traffic condition and a larger packet size degrade the maximum throughput. The result for $\overline{B L}=1$ is almost same as that for output unbalanced traffic (see Figure 9). On the other hand, the result for $\overline{B L}=10$ show higher performance than that of output unbalanced traffic (see Figure 10). This is because unbalanced traffic at input ports causes less HOL blocking than at output ports.

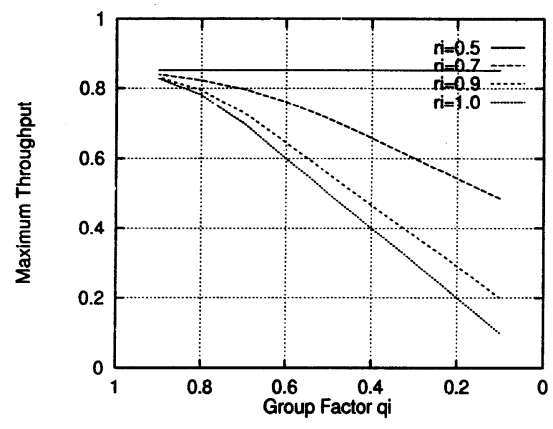

Figure 11 Unbalanced traffic at input ports $\left(N_{O}=10, \overline{B L}=1\right)$.

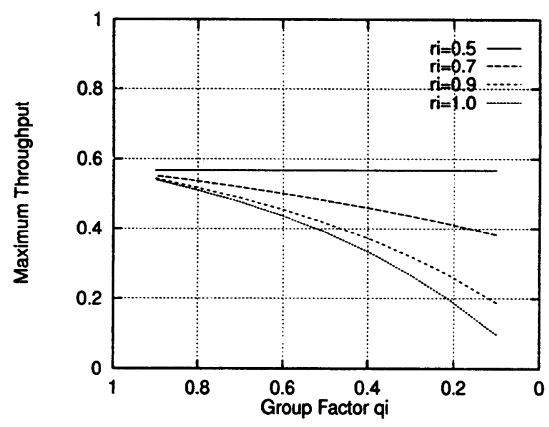

Figure 12 Unbalanced traffic at input ports $\left(N_{O}=10, \overline{B L}=10\right)$.

\subsection{Case of Mixture with Stream Traffic}

Finally, we derive the maximum throughput in the case where the bursty traffic and the stream traffic coexist. Here, we assume that the stream traffic occupies some portion of the link with 
constant peak rate. For example, this class of traffic can support an uncompressed video transfer service.

Let $R$ denote the peak rate of stream traffic normalized by the link capacity. The switch can simultaneously accept $m(\leq\lfloor 1 / R\rfloor)$ calls of stream traffic. We assume that call arrivals of the stream traffic follow a Poisson distribution with mean $\lambda_{C B R}$, and its service time (or call holding time) does an exponential distribution with mean $1 / \mu_{C B R}$. While both bursty and stream traffic share a link, cells from the stream traffic are given a higher priority. Namely, cells from stream traffic arriving at the input port are transferred to its destined output port prior to cells from bursty traffic [RFM94]. By this control mechanism, it can be considered that bursty traffic can utilize $1-n R$ of the link capacity when $n$ calls of stream traffic are accepted. We note here that if compressed video transfer service is accommodated as stream traffic, a more capacity can be utilized by the bursty traffic. Thus, the maximum throughput derived in the below should be regarded as the "minimum" guaranteed throughput for the bursty traffic.

Since the stream traffic is given a high priority, it can be modeled by a $M / M / m / m$ queueing system. By letting $\pi_{n}$ be the probability that $n$ calls of stream traffic are accepted in steady state, we have (e.g., [BG87])

$\pi_{n}=\left[\sum_{n=0}^{m}\left(\frac{\lambda_{C B R}}{\mu_{C B R}}\right)^{n} \frac{1}{n !}\right]^{-1}\left(\frac{\lambda_{C B R}}{\mu_{C B R}}\right)^{n} \frac{1}{n !}$.

Since the service time of steam traffic can be assumed to be much longer than cell or the packet transmission time of bursty traffic, an available link capacity to bursty traffic is regarded to be constant when the number of accepted calls of stream traffic is fixed. By letting $\rho_{n}$ be the maximum throughput for bursty traffic when $n$ calls of the stream traffic are accepted, we have [RFM94]

$\rho_{n}=(1-n R) \rho$,

where $\rho$ is defined as the maximum throughput of bursty traffic when all link capacity is allocated to bursty traffic, and has been already derived in Subsection 4.1. Consequently, the "averaged" maximum throughput $\rho^{\prime}$ is obtained as

$\rho^{\prime}=\sum_{n=0}^{m} \pi_{n} \rho_{n}$

Figure 13 shows the maximum throughput of bursty traffic and stream dependent on an offered traffic load for stream traffic for $N_{O}=50, \mu_{C B R}=0.1, R=0.2$ and $m=5$. From this figure, we can observe the natural idea that the larger the average packet length is, the smaller the maximum allowable throughput of bursty traffic is. Therefore, the available bandwidth allocated to the stream traffic should be limited in some way to avoid a degradation of bursty traffic efficiency to some degree. One possible approach is to decrease $m$, the maximum number of calls of stream traffic that the switch can accept. In Figure 14, the maximum throughput of both bursty traffic and stream traffic dependent on the offered traffic load for stream traffic for $\overline{B L}=1$ and several values of $m$. It shows that the performance degradation of bursty traffic can be avoided to some extent by limiting $m$. 


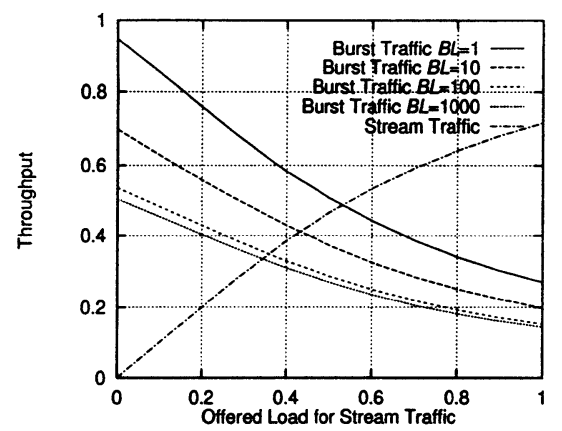

Figure 13 Throughput vs. the offered load of stream traffic.

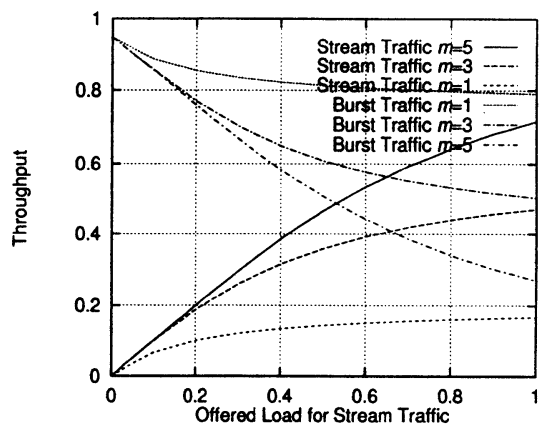

Figure 14 Effect of an available link capacity limitation on stream traffic.

\section{CONCLUSION}

In this paper, an ATM switch with input and output buffer equipped with back-pressure function was treated. We have analyzed its performance under bursty traffic condition for applying it to ATM LANs. We have derived the maximum throughput under the assumption that the switch size is infinite. Consequently, we have shown that larger packet lengths drastically degrade the performance of the switch. However, it is possible to sustain such a degradation to some extent by larger output buffers. At least, the output buffer size comparable to the average packet length is necessary to gain a sufficient performance. We have already analyzed the packet delay distribution and the approximate packet loss probability of the switch. These results can be found in [OWMM95].

Last, we note that our analytic approach described in the current paper can be applied to other cases, e.g., the case where the switching speed is $L(1 \leq L \leq N)$ times faster than the link speed (see, e.g., [YMKH92]), or the case where, when $L^{\prime}(>L)$ cells are simultaneously destined for the same output buffer, $\left(L^{\prime}-L\right)$ cells are lost or kept awaiting at the input buffer.

For further works, we should evaluate the performance of the network in which two or more ATM switches are interconnected. In such a network, even when a long term congestion introduces large queue length at the input buffer, cell losses may be avoided to send a back-pressure signal to the upper adjacent switches.

\section{ACKNOWLEDGMENT}

We would like to thank Dr. Hiroshi Suzuki and Dr. Ruixue Fan with NEC Corporation, C\&C System Laboratories, for their invaluable suggestions.

\section{REFERENCES}

[BG87] Dimitri Bertsekas and Robert Gallager. Data Networks. Prentice-Hall, Englewood Cliffs, New Jersey, 1987. 
[Ili90] Ilias Iliadis. Head of the line arbitration of packet switches with input and output queueing. In Fourth International Conference on Data Communication Systems and their Performance, pages 85-98, Barcelona, Spain, June 1990.

[Ili92a] Ilias Iliadis. Performance of a packet switch with input and output queueing under unbalanced traffic. In Proceedings of IEEE INFOCOM '92, volume 2, pages 743-752 (5D.4), Florence, Italy, May 1992.

[Ili92b] Ilias Iliadis. Synchronous versus asynchronous operation of a packet switch with combined input and output queueing. Performance Evaluation, (16):241-250, 1992.

[KHM86] Mark J. Karol, Michael G. Hluchyj, and Samuel P. Morgan. Input vs. output queueing on a space-division packet switch. In Proceedings of IEEE GLOBECOM '86, pages 659-665, Houston, Texas, December 1986.

[OWMM95] Hiroyuki Ohsaki, Naoki Wakamiya, Masayuki Murata, and Hideo Miyahara. Performance of an input/output buffered type ATM LAN switch with back-pressure function. submitted to IEEE Transactions on Networking, 1995.

[RFM94] Kenji Yamada Ruixue Fan, Hiroshi Suzuki and Noritaka Matsuura. Expandable ATOM switch architecture (XATOM) for ATM lans. ICC'94, 51994.

[WHPV88] Saul A. Teukolsky William H. Press, Brian P. Flannery and William T. Vetterling. Numerical Recipes in C. Cambridge University Press, 1988.

[YMKH92] Yuji Oie, Masayuki Murata, Koji Kubota, and Hideo Miyahara. Performance analysis of nonblocking packet switches with input / output buffers. IEEE Transactions on Communications, 40(8):1294-1297, August 1992. 